\title{
Chemopreventive efficacy of salvianolic acid B phospholipid complex loaded nanoparticles against experimental oral carcinogenesis: implication of sustained drug release
}

\author{
Wei Liu ${ }^{1,2}$, Zengtong Zhou ${ }^{3}$, Laikuan $\mathrm{Zhu}^{4}$, Hongquan $\mathrm{Li}^{5}$, Lan $\mathrm{Wu}^{3} \wedge$ \\ ${ }^{1}$ Department of Oral and Maxillofacial-Head and Neck Oncology, Fengcheng Hospital, Shanghai, China; ${ }^{2}$ Department of Oral and Maxillofacial- \\ Head and Neck Oncology, Shanghai Ninth People's Hospital, Shanghai Jiao Tong University School of Medicine, Shanghai, China; ${ }^{3}$ Department of \\ Oral Mucosal Diseases, Shanghai Ninth People's Hospital, Shanghai Jiao Tong University School of Medicine, College of Stomatology, Shanghai Jiao \\ Tong University, National Center for Stomatology, National Clinical Research Center for Oral Diseases, Shanghai Key Laboratory of Stomatology, \\ Shanghai, China; ${ }^{4}$ Department of Endodontics and Operative Dentistry, Shanghai Ninth People's Hospital, Shanghai Jiao Tong University School of \\ Medicine, Shanghai, China; ${ }^{5}$ Department of Oral Surgery, Shanghai Xuhui District Dental Disease Center, Shanghai, China \\ Contributions: (I) Conception and design: L Wu, W Liu; (II) Administrative support: Z Zhou, L Zhu; (III) Provision of study materials or patients: \\ H Li, W Liu; (IV) Collection and assembly of data: H Li, W Liu; (V) Data analysis and interpretation: H Li, L Zhu; (VI) Manuscript writing: All \\ authors; (VII) Final approval of manuscript: All authors. \\ Correspondence to: Dr. Hongquan Li. Department of Oral Surgery, Shanghai Xuhui District Dental Disease Center, 500 Fenglin Road, Shanghai \\ 200031, China. Email: lihq8092@163.com; Dr. Laikuan Zhu. Department of Endodontics and Operative Dentistry, Shanghai Ninth People's \\ Hospital, 500 Quxi Road, Shanghai 200011, China. Email: zhulk1997@163.com; Dr. Lan Wu. Department of Oral Mucosal Diseases, Shanghai \\ Ninth People’s Hospital, 500 Quxi Road, Shanghai 200011, China. Email: teana_wu@sina.com.
}

Background: Although we have previously demonstrated that phospholipid complex loaded nanoparticles (PLC-NPs) encapsulating salvianolic acid B (SAB) can enhance anticancer activity in head and neck cancer and precancerous cells in vitro, the chemopreventive efficacy of SAB-PLC-NPs (nano-SAB) in vivo remains unclear. Here, we aimed to investigate the in vivo efficacy of nano-SAB against experimental oral carcinogenesis.

Methods: Oral tongue carcinogenesis was induced in C57BL/6 mice through the administration of 4-nitroquinoline-N-oxide (4NQO, $100 \mu \mathrm{g} / \mathrm{mL}$ ) in drinking water for 22 weeks. To preliminarily evaluate the effect of sustained drug release against oral carcinogenesis, free- or nano-SAB $(16.6 \mathrm{mg} / \mathrm{kg} / \mathrm{d}) \mathrm{was}$ administered orally for 18 weeks, and the treatment was discontinued for the remaining 4 weeks.

Results: Histological evaluation revealed a significant $(\mathrm{P}<0.05)$ decrease in the incidence of carcinoma in free-SAB-treated (16.7\%) and nano-SAB-treated (10.0\%) mice compared to mice exposed to $4 \mathrm{NQO}$ alone $(34.3 \%)$. A decrease in carcinoma growth rate was also observed in free-SAB-treated (12.2\%) and nanoSAB-treated (5.5\%) mice compared to the 4NQO-exposed group (18.3\%), even after drug withdrawal for 4 weeks. Immunohistochemical analysis revealed that nano-SAB treatment effectively suppressed Ki-67, proliferative cell nuclear antigen (PCNA), and cyclin D1 expression in high-risk dysplastic lesions compared to free-SAB-treated and 4NQO-exposed groups (all $\mathrm{P}<0.05$ ). Importantly, nano-SAB maintained low levels of Ki-67, PCNA, and cyclin D1 expression even after drug withdrawal for 4 weeks.

Conclusions: Together with our previous in vitro data, this in vivo study confirms that nano-SAB has superior chemopreventive efficacy by promoting more potent anti-proliferation and cell cycle arrest responses. These findings demonstrate the potential of SAB-PLC-NPs as promising chemopreventive agents for treating oral carcinogenesis.

Keywords: Salvianolic acid B (SAB); phospholipid nanoparticles; cell proliferation; oral leukoplakia; squamous cell carcinoma

^ ORCID: 0000-0001-8286-1063. 
Submitted Aug 24, 2021. Accepted for publication Dec 24, 2021.

doi: $10.21037 /$ atm-21-4457

View this article at: https://dx.doi.org/10.21037/atm-21-4457

\section{Introduction}

Cancers of the lip and oral cavity represent the 16th most common neoplasm worldwide, with almost 355,000 new diagnoses and over 177,000 deaths estimated in 2018 (1). Oral squamous cell carcinoma (OSCC) accounts for over $90 \%$ of oral cancer and often develop from precancerous lesions. Early prevention and intervention of OSCC development are of utmost importance to reduce the incidence and mortality of this disease $(1,2)$. Oral carcinogenesis is a multi-step cumulative process that is associated with the accumulation of genetic alterations over time as a result of chronic exposure to carcinogens such as tobacco and cigarette smoke $(3,4)$. The multiple stages associated with OSCC progression indicate that a prevention strategy that can delay, inhibit, or reverse carcinogenesis before it becomes an invasive disease would be the most beneficial approach (5). Using phytochemicals as a chemoprevention agent would be a potentially useful strategy to delay or block the carcinogenic process and oral cancer development $(6,7)$. The promising phytochemicals, such as green tea extract, curcumin, genistein, resveratrol, lycopene, black raspberries, and canthaxanthin, for oral cancer chemoprevention have been summarized in recent review articles $(6,7)$.

Our previous study found that salvianolic acid B (SAB) phospholipid complex (PLC) loaded-nanoparticles (NPs) may be a promising chemopreventive agent for head and neck cancer cells, as well as precancerous oral leukoplakia cells in vitro (8). To overcome the limitations of low bioavailability and poor systemic delivery of $\mathrm{SAB}$, we previously described the use of PLC-NPs encapsulating SAB as a potential carrier for intervention of OSCC and precancerous cells (8). SAB-PLC-NPs (nano-SAB) were found to be significantly more potent than free-SAB in increasing anti-proliferative bioactivity and inducing cell apoptosis and cell cycle arrest (8). In particular, we found that enhanced cellular uptake of $\mathrm{SAB}$ by nano-formulation led to increased anticancer potential, presumably by increasing drug bioavailability (8). Based on the findings of our previous in vitro study, the current study was carried out to determine the chemopreventive efficacy of nano-SAB in vivo.

The 4-nitroquinoline-N-oxide (4NQO)-induced mouse model of oral tongue carcinogenesis is an excellent in vivo model for studying the early multi-step events in oral carcinogenesis development that also allows for the chemopreventive potential of natural and synthetic agents to be examined (9). As a synthetic water-soluble chemical carcinogen, 4NQO mimics chronic tobacco exposure resulting in histological and molecular alterations similar to human oral carcinogenesis (10). Histologically, the 4NQOinduced mouse model has sequential stages of oral epithelial lesions resembling the multistage carcinogenesis of human oral carcinogenesis, including epithelial hyperplasia, various degrees of dysplasia, and OSCC (10). Emerging studies have suggested that this animal model may provide a predictable preclinical strategy to mimic human oral carcinogenesis, which can be used to investigate the efficacy of novel chemical compounds for OSCC chemoprevention (9-11).

In the current study, we conducted a preclinical prevention trial in the $4 \mathrm{NQO}$-induced oral carcinogenesis mouse model to determine the chemopreventive efficacy of nano-SAB against multistage development of OSCC. C57BL/6 mice were administered nano-SAB concurrently with $4 \mathrm{NQO}$ exposure to evaluate the effect of nano-SAB on disease initiation and progression of OSCC through histological and immunohistochemical evaluation of $\mathrm{Ki}-67$, proliferative cell nuclear antigen (PCNA), cyclin D1, and p16 expression. Since we previously demonstrated that nano-formulation can enhance the cellular uptake of $\mathrm{SAB}$ and improved its bioavailability in vitro (8), we also sought to determine whether nano-SAB could enhance sustained drug release after accumulation in vivo for 18 weeks by discontinuing treatment with free- and nano-SAB for the final 4 weeks (weeks 19-22) of the study.

We present the following article in accordance with the ARRIVE reporting checklist (available at https://atm. amegroups.com/article/view/10.21037/atm-21-4457/rc).

\section{Methods}

\section{Reagents, animals, and study design}

SAB and nano-formulated SAB-PLC-NPs were provided by Peng and colleagues $(12,13)$, as mentioned in our previous in vitro study (8). 4NQO (Sigma-Aldrich, St. Louis, MO, USA) was added to the drinking water to a 
final concentration of $100 \mu \mathrm{g} / \mathrm{mL}$. Six-week-old female C57BL/6J mice (SPF, SCXK2012-0002) were obtained from Shanghai Slac Laboratory Animal Co. Ltd., China. The mice were housed under standard conditions of temperature and humidity with an alternating 12 -h light-dark cycle and were fed a normal pellet diet and water. A protocol was prepared before the study without registration. Experiments were performed under a project license (No. SH9H-2020-A85-1) granted by the Animal Ethical Committee of Shanghai Ninth People's Hospital, in compliance with internationally recognized and institutional guidelines for the care and use of animals.

After 1 week of acclimatization, the mice were randomly divided by computer-generated randomization numbers into 6 groups (Figure 1A): 4NQO alone (Group A, n=35), 4NQO + SAB (Group B, n=30), 4NQO + SAB-PLC-NPs (Group C, $n=30$ ), normal water control (Group D, n=5), normal water + SAB control (Group E, $\mathrm{n}=5$ ), and normal water + SAB-PLC-NPs control (Group $\mathrm{F}, \mathrm{n}=5$ ). The mice in groups A-C were given 4NQO $(100 \mu \mathrm{g} / \mathrm{mL})$ in drinking water, while mice in groups $\mathrm{D}-\mathrm{F}$ received normal drinking water for 22 consecutive weeks. Meanwhile, groups B and C mice were administered free- and nano-SAB (both $16.6 \mathrm{mg} / \mathrm{kg} / \mathrm{d}$ ) orally by gavage concurrent with $4 \mathrm{NQO}$ exposure. To determine whether nano-formulation could enhance sustained drug release after accumulation for 18 weeks, treatment with free- and nano-SAB was discontinued in groups B and C for the final 4 weeks (weeks 19-22) of the study. Groups D, E, and F mice were administered normal drinking water, and free- and nano-SAB as controls, respectively.

The drug concentration of free- and nano-SAB $(16.6 \mathrm{mg} / \mathrm{kg}$ ) received by $\mathrm{C} 57 \mathrm{BL} / 6 \mathrm{~J}$ mice in the current study was based on the equivalent dosage of SAB administered to male Syrian hamsters $(10 \mathrm{mg} / \mathrm{kg})$ in previous studies $(14,15)$. The mice in each group were evaluated weekly for bodyweight, and all mice underwent a biweekly full oral cavity macroscopic examination. To determine the impact of multistage intervention on the activity of free- and nano-SAB against oral carcinogenesis, 7-10 mice in groups A-C were euthanized for tongue tissue retrieval at weeks $8,14,18$, and 22. Tissue samples were collected at the indicated time points for histopathological and immunochemical examinations.

\section{Histopatbology and immunobistochemistry (IHC)}

Whole tongue specimens were fixed in formalin, embedded in paraffin, and processed for routine histopathological examination from hematoxylin and eosin $(\mathrm{H} \& \mathrm{E})$ staining sections. The diagnoses of oral lesions with epithelial hyperplasia, various degrees of dysplasia, and carcinoma were made based on the World Health Organization criteria (16) and recommended in previous reports on the C57BL/6J mouse oral cancer model $(17,18)$. The worst histological grade was identified and diagnosed by an oral pathologist blinded to treatment groups. The primary outcome of preventive efficacy was measured by cumulative incidence of oral dysplasia/carcinoma in the 18-week trial of experimental groups A-C. Incidence of dysplasia/carcinoma was reported as the percentage of animals based on the worst histology, and cumulative incidence of dysplasia/ carcinoma was calculated by summing up individual mice at the indicated time points and reporting as the total incidence of dysplasia/carcinoma per group.

Five-micrometer-thick tissue sections were further processed for IHC analysis. Sections were stained with monoclonal antibodies against Ki67 (Ab16667, 1:100) and PCNA (Ab92729, 1:50) as cell proliferation markers and against cyclin D1 (Ab134175, 1:10) and p16 (Ab54210, 1:500) as cell cycle markers. PCNA plays an important role in cell proliferation and cell cycle regulation, while $\mathrm{Ki}-67$ is detected throughout all phases of the cell cycle with the exception of early G1 phase. Cyclin D1 is a nuclear protein involved in the G1/S transition of the cell cycle, while p16 is a regulator of the G1 phase of the cell cycle (19). These antibodies were obtained from Abcam, Cambridge, UK. IHC was performed using the Leica Bond Max automated stainer (Leica Biosystems, Weztlar, Germany) according to the manufacturer's protocol in the Department of Oral Pathology, Shanghai Ninth People's Hospital, Shanghai, China. All slides were stained using previously optimized conditions with appropriate positive and negative controls. The quantitative evaluation of immunoreactivity was performed according to the criteria of staining intensities described previously (20). Briefly, 5 digital images of highpower fields at $400 \times$ magnification selected randomly on immunostained sections were captured by the DP 70 CCD camera (Olympus, Tokyo, Japan). Then, positive cells were counted using Image Pro-Plus software (version 6.0, Media Cybernetics, Rockville, MD, USA) in this study.

\section{Statistical analysis}

The objective of this study was to evaluate the efficacy of nano-SAB compared with free-SAB against oral carcinogenesis. Hence, the statistical analysis of each group 
A

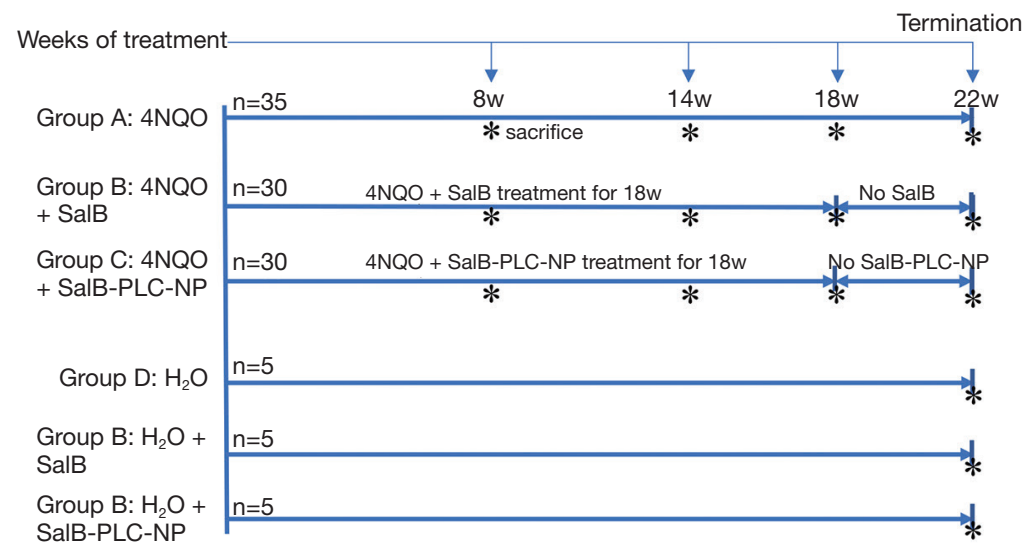

B

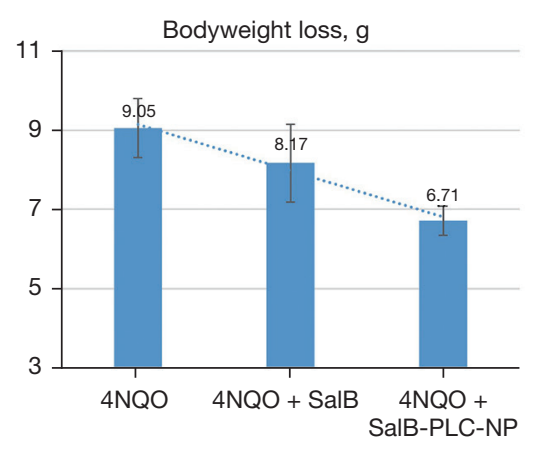

D

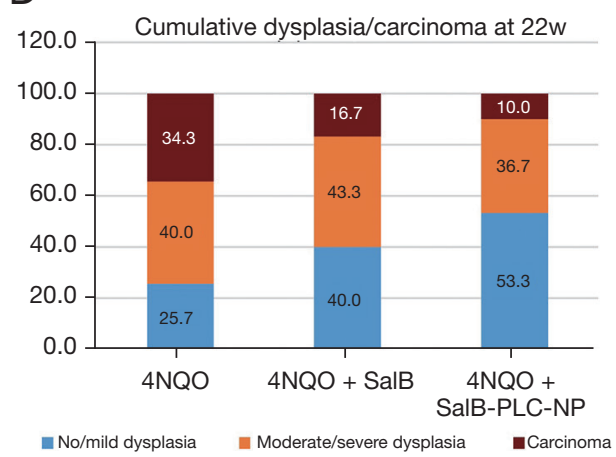

C

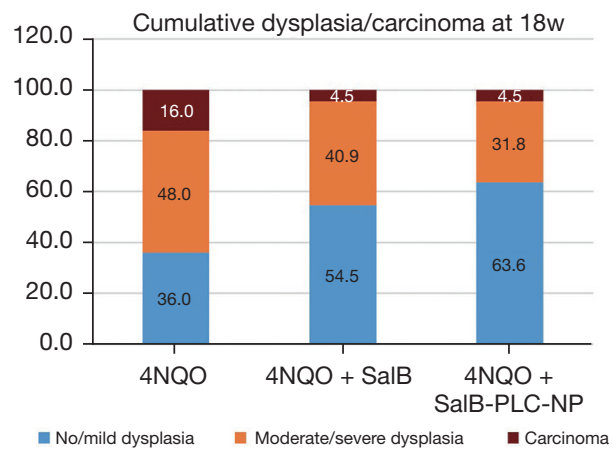

E

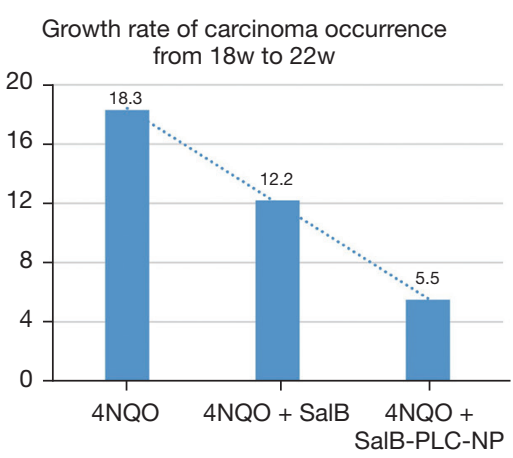

Figure 1 Chemoprevention in mice oral carcinogenesis. (A) Experimental protocol of the 6 groups. (B) Bodyweight changes in the experimental groups. Cumulative incidence of oral dysplasia/carcinoma (C) at week 18 and (D) at week 22. (E) Growth rate of carcinoma occurrence in experimental groups after the drug withdrawal for 4 weeks. The asterisk $\left(^{*}\right)$ indicates that the mice were sacrificed at the indicated time points (weeks 8, 14, 18, or 22). NQO, 4-nitroquinoline-N-oxide; SalB, salvianolic acid B; PLC-NP, phospholipid complex loaded nanoparticles.

was mainly performed with reference to the result of the nano-SAB group. Quantitative variables are presented as mean \pm standard deviation $(\mathrm{SD})$. Differences in quantitative variables among 2 and 3 groups were calculated by the Student's $t$-test and one-way analysis of variance (ANOVA), respectively. If the quantitative variables did not follow a normal Gaussian distribution, the differences between 2 groups were calculated using a Mann-Whitney $U$ test. Differences in qualitative variables were calculated by the $\chi^{2}$ or Fisher's exact test. A 2 -sided $\mathrm{P}<0.05$ was considered 
statistically significant. Statistical analysis was performed using the SPSS 21.0 software (IBM SPSS Inc.).

\section{Results}

\section{Effect of nano-SAB on $4 \mathrm{NQO}$-induced oral carcinogenesis}

To investigate the effect of nano-SAB on the general body response to the carcinogen, $4 \mathrm{NQO}$, we determined the bodyweight changes (maximum minus minimum) in the 22-week trial of the experimental and control groups. The bodyweight of mice was found to be very similar at the beginning of the experiment in the various groups A-F. After the completion of treatment at week 22, the mean bodyweight of groups A-C (Figure $1 B$ ) showed a significant $(\mathrm{P}<0.05$, one-way ANOVA) gradual decrease from the 4NQO-exposed group $(9.05 \pm 0.74 \mathrm{~g})$ to the freeSAB-treated $(8.17 \pm 0.98 \mathrm{~g})$ and nano-SAB-treated groups $(6.71 \pm 0.37 \mathrm{~g})$. Conversely, the mean bodyweight of groups D-F showed a similar normal increase between the normal drinking water $(7.08 \pm 0.17 \mathrm{~g})$, free-SAB control $(7.17 \pm 1.08 \mathrm{~g})$, and nano-SAB control $(6.98 \pm 1.36 \mathrm{~g})$ groups. Bodyweight and histological changes of the experimental groups are presented in Table S1.

To assess the chemopreventive effect of nano-SAB on the multistage development of OSCC, we examined the cumulative incidence of oral dysplasia/carcinoma in the 18-week trial of experimental groups A-C. After the completion of treatment at week 18 , the incidence of carcinoma $(16.0 \%)$ in the $4 \mathrm{NQO}$-exposed group was higher than that observed in the free-SAB treated and nano-SAB treated groups (both $4.5 \%$ ) (Figure 1C). The incidence of moderate dysplasia or worse in groups A-C showed a decreasing trend $(\mathrm{P}=0.059$, one-way ANOVA) from the 4NQO-exposed group (64.0\%) to the free-SABtreated $(45.5 \%)$ and nano-SAB-treated $(36.3 \%)$ groups. No histological abnormalities were found in the normal control, or the free-SAB and nano-SAB control mice (groups D-F). Furthermore, no clinical signs of toxicity were observed in the drug control mice (groups E, F) throughout the whole trial.

To evaluate the effect of sustained drug release after 18 -week accumulation of nano-SAB on oral carcinogenesis, we focused on the cumulative incidence of oral dysplasia/ carcinoma in the 22-week trial of experimental groups A-C (Figure 1D). After the completion of $4 \mathrm{NQO}$ treatment at week 22, the incidence of carcinoma was significantly lower $\left(\mathrm{P}<0.05, \chi^{2}\right.$ test $)$ in the free-SAB $(16.7 \%)$ and nano-SAB
(10.0\%) groups than in the 4NQO-exposed group (34.3\%). Consistent with these findings, the incidence of moderate dysplasia or worse in groups A-C was significantly lower $\left(\mathrm{P}<0.05, \chi^{2}\right.$ test $)$ in the free-SAB $(60.0 \%)$ and nano-SAB $(46.7 \%)$ groups than in the $4 \mathrm{NQO}$-exposed group $(74.3 \%)$. Interestingly, the carcinoma growth rate in groups A-C (Figure 1E) was higher in the 4NQO-exposed group (18.3\%) than in the free-SAB $(12.2 \%)$ and nano-SAB $(5.5 \%)$ groups, even after withdrawal of the drug for the final 4 weeks of the study. Representative macroscopic lesions and microscopic histological images are shown in Figure 2.

\section{Effect of nano-SAB on cell proliferation and cell cycle markers}

To explore the effect of nano-SAB on 4NQO-induced cell proliferation and cell cycle progression in oral tongue carcinogenesis, we analyzed the expression of well-defined cell proliferation (Ki67 and PCNA) and cell cycle (cyclin D1 and p16) markers. Representative immunohistochemical images of normal mucosa, dysplasia, and squamous cell carcinoma are shown in Figure 3. Since moderate/severe dysplasia represents the high-risk potentially malignant phase, we focused on the effect of SAB on the expression of cell proliferation and cell cycle markers in this phase to determine the chemopreventive potential of nano-formulation.

At week 18, after the completion of $4 \mathrm{NQO}$ treatment, 5,5 , and 4 high-risk dysplastic lesions were observed in the experimental groups A, B, and C, respectively. Overexpression of Ki-67, PCNA, and cyclin D1 was found in the 4NQO-exposed mice (group A), but their expression patterns were significantly decreased (all $\mathrm{P}<0.05$ ) in nano-SAB-treated mice (group C) compared to free$\mathrm{SAB}$ treated mice (group B). In contrast, p16 was weakly expressed in group A, with increased expression in group C compared to group B (data not shown). At week 22 of $4 \mathrm{NQO}$ treatment following drug withdrawal for weeks 1922, 2, 4, and 4 high-risk dysplastic lesions were observed in the experimental groups $\mathrm{A}, \mathrm{B}$, and $\mathrm{C}$, respectively. The expression trends of Ki-67, PCNA, and cyclin D1 observed at week 22 were similar to those observed at week 18 in groups A, B, to C, respectively (Figure 4).

To evaluate the implication of sustained drug release after withdrawal (weeks 19-22) of nano-SAB on cell proliferation and cell cycle markers, we focused on the differences in the expression patterns of these markers at week 18 versus week 22 (Figure 4). The expression levels of Ki-67, PCNA, and cyclin D1 in groups A and B were significantly higher 
A

B
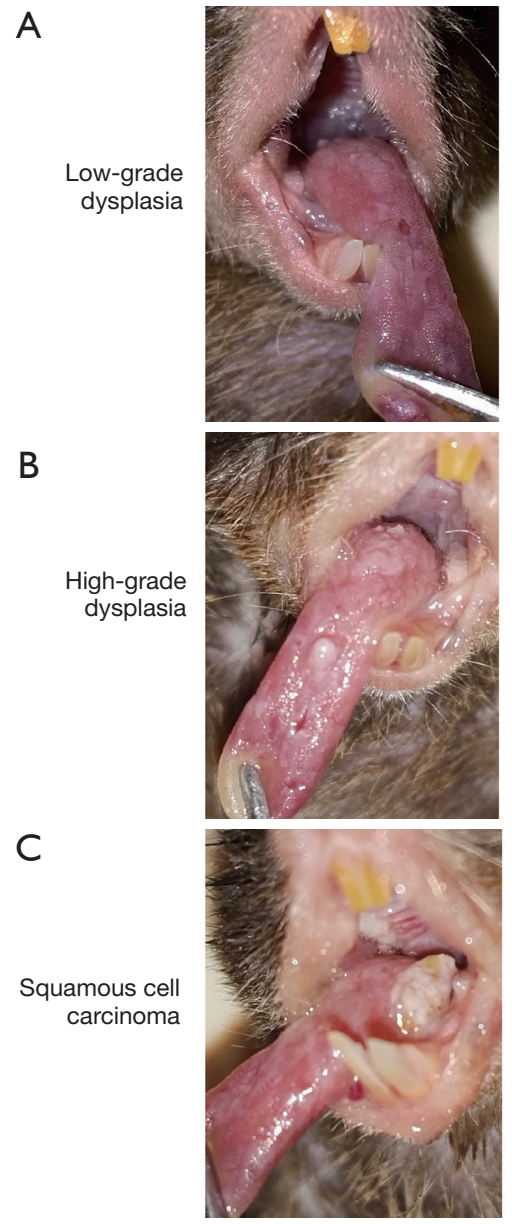
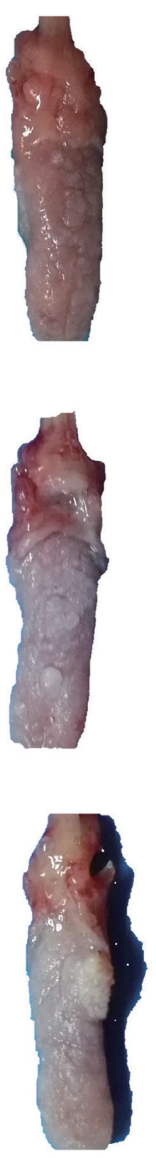
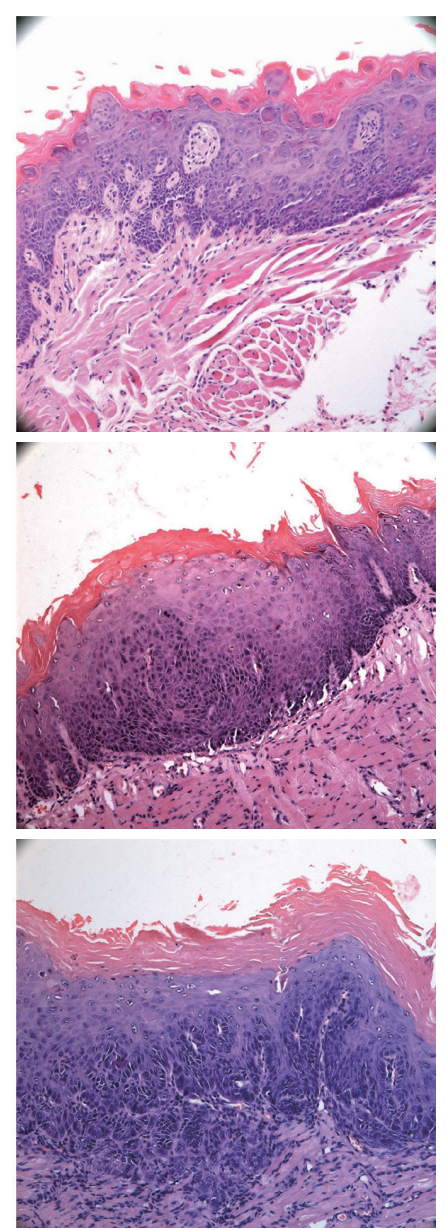

Figure 2 Representative macroscopic lesions and microscopic histologic images (hematoxylin-eosin staining; magnification, $\times 100$ ) of mice oral carcinogenesis. (A) Low-risk (mild) dysplasia, (B) high-risk (moderate/severe) dysplasia, and (C) squamous cell carcinoma.

at week 22 than week 18 (all $\mathrm{P}<0.05, t$-test). Interestingly, no significant differences in Ki-67, PCNA, or cyclin D1 expression levels between the 2 time points were observed in group $\mathrm{C}(\mathrm{P}>0.05, t$-test $)$, which displayed significantly weaker expression levels than did groups A and B (all $\mathrm{P}<0.05, t$-test) at the same time points. In addition, free$\mathrm{SAB}$ or nano-SAB alone-administrated mice (groups $\mathrm{E}$ and F) displayed no significant differences in cell proliferation or cell cycle marker expression compared to the normal control (group D).

\section{Discussion}

Chemoprevention is defined as the use of natural, synthetic, or biological compounds to inhibit, prevent, or reverse the initial phases of carcinogenesis or the progression of neoplastic cells to cancer (6). We and others have demonstrated that SAB has significant potential to be used for oral cancer chemoprevention due to its effectiveness both in vitro and in the xenograft mice model $(21,22)$, as well as in the 7,12-dimethlybenz(a)anthracene (DMBA)induced hamster cheek pouch model $(14,15)$. The DMBAinduced hamster cheek pouch and 4NQO-induced mouse tongue cancer models are two classical animal models of oral carcinogenesis (9-11). However, the DMBA-induced model is a questionable surrogate for the human oral cavity, due to the cheek pouch, which is specifically designed for the storage and transport of food in hamsters. Moreover, DMBA is not found in tobacco smoke or the environment but is an oxidatively activated carcinogen. In contrast, 4NQO causes damage similar to the carcinogens in tobacco and induces molecular and pathological changes similar to human OSCC (9-11). Hence, we used the 4NQO-induced mouse oral carcinogenesis in this study. 


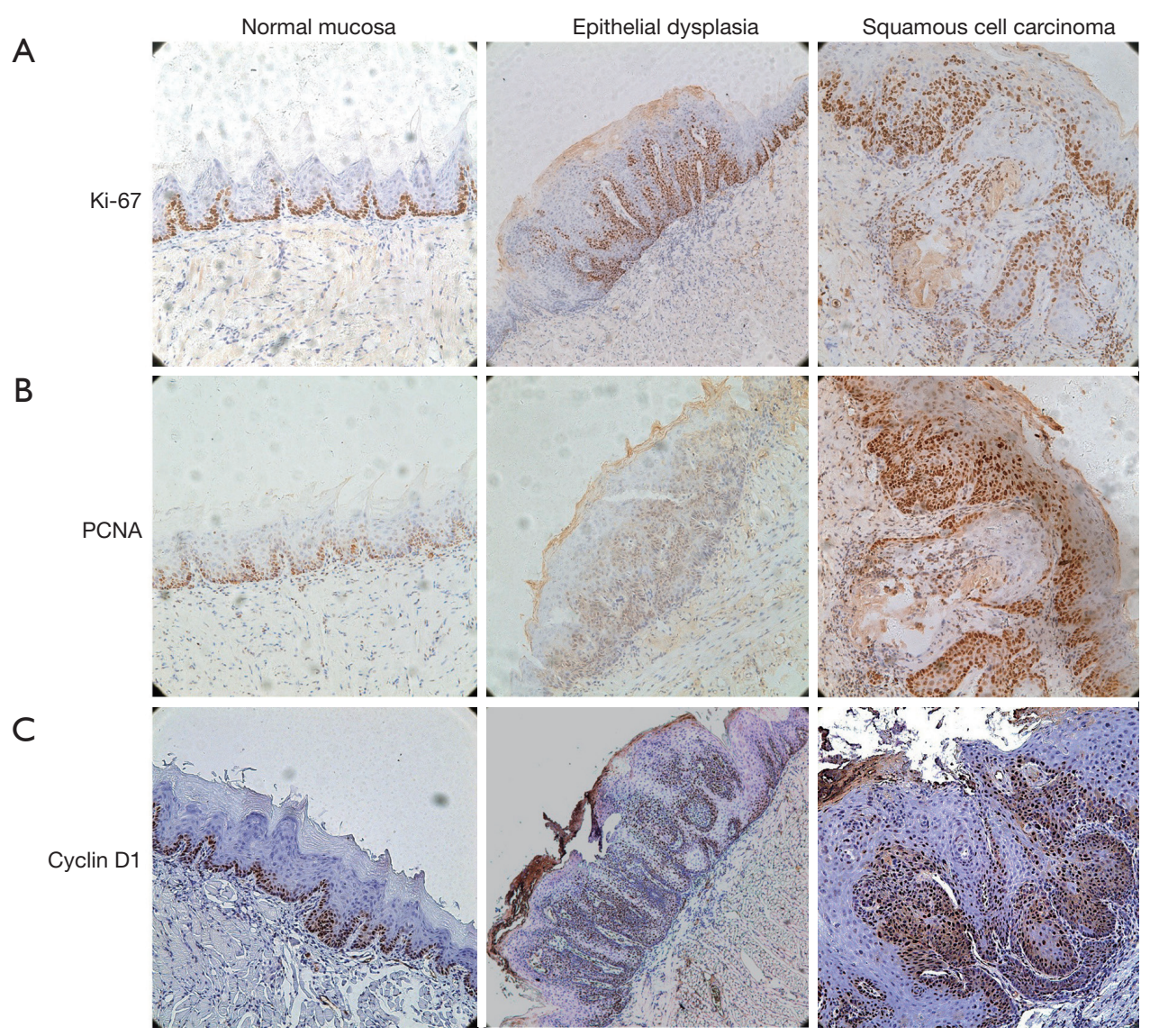

Figure 3 Representative immunohistochemical images (streptavidin-peroxidase method; magnification, $\times 100$ ) of normal mucosa, dysplasia, and squamous cell carcinoma in mice oral carcinogenesis. (A) Ki67, (B) proliferative cell nuclear antigen (PCNA), and (C) Cyclin D1.
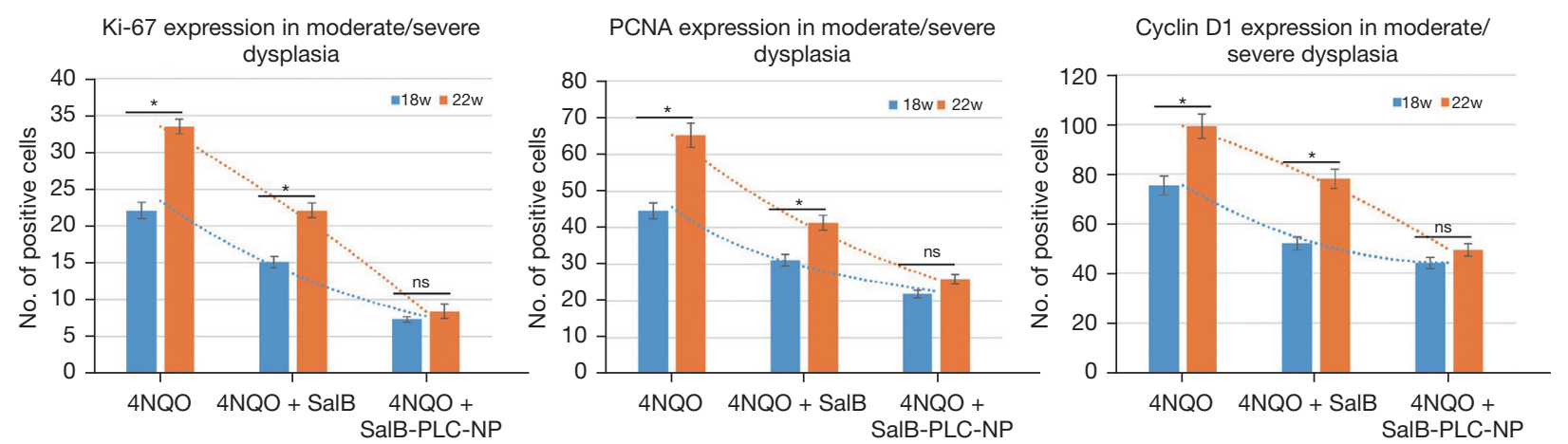

Figure 4 Expression levels of Ki67, PCNA, and cyclin D1 in moderate/severe dysplasia of experimental groups at week 18 versus week $22 . *$, $\mathrm{P}<0.05$; ns. not significant.

Nanochemoprevention is a concept in which nanotechnology-based regimens are developed for cancer chemoprevention (23). Nanoparticle-mediated delivery is a promising approach to enhance bioavailability and increase the efficacy of several chemopreventive agents for OSCC (24-28). Our previous study clearly demonstrated that nano$\mathrm{SAB}$ can enhance cellular uptake and anticancer efficacy in head and neck cancer cells, as well as in precancerous 
cells in vitro (8). The current in vivo study aimed to further investigate the chemopreventive efficacy and sustained drug release of nano-SAB in 4NQO-induced C57BL/6 mouse oral tongue carcinogenesis by evaluating its antiproliferative and cell cycle arrest effects through histological studies. Overall, the chemopreventive efficacy of SAB in the 4NQO-induced mouse oral tongue model was in agreement with findings in the DMBA-induced hamster cheek pouch model $(14,15)$. Bodyweight loss is a common phenomenon in tumor-bearing animals, usually because the continuous demand of energy for cancer cells depletes energy reserves. Here, a drastic weight loss was observed in mice exposed to $4 \mathrm{NQO}$ alone. However, the weight loss observed in the nano-SAB-treated group was less than that in freeSAB- and 4NQO-treated mice (Figure $1 B$ ). These findings suggest that nano-SAB treatment prevented the depletion of energy reserves by cancer cells, thereby reducing the enhanced metabolic rate, which causes weight loss in the other 4NQO-exposed groups.

Importantly, the incidence of high-risk dysplasia and carcinoma was reduced after nano-SAB treatment compared to free-SAB treatment, suggesting that a superior efficacy of $\mathrm{SAB}$ chemoprevention was realized through nanoformulated PLC-NPs, leading to either the inhibition or prevention of malignant transformation of oral dysplasia. Moreover, high-risk dysplastic lesions in 4NQO-exposed mice showed a substantial increase in Ki-67, PCNA, and cyclin D1 expression, whereas SAB treatment, particular nano-formulation, effectively suppressed the expression of these proteins in 4NQO-induced mice (Figure 3D). $\mathrm{Ki}-67, \mathrm{PCNA}$, and cyclin D1 are major cell proliferative markers used to examine cell cycle progression and tumor development. Thus, decreased expression of these markers indicates the suppression of cancer development. These results are consistent with our previous in vitro study, which showed that nano-SAB was significantly more potent than free-SAB in inhibiting proliferation and inducing cell cycle arrest (8). Our findings suggested that nano-SAB may exert its superior efficacy of chemoprevention by exhibiting more potent anti-proliferation effects and inducing cell cycle arrest through the blockade of Ki-67, PCNA, and cyclin D1 expression.

We also explored the implication of sustained drug release on oral carcinogenesis after 18 -week accumulation of nano-SAB by discontinuing treatment with free- and nano-SAB for the final 4 weeks (weeks 19-22) of the study. After drug withdrawal for 4 weeks, the incidence of carcinoma and high-risk dysplasia was significantly lower in nano-SAB-treated mice (group $\mathrm{C}$ ) than in mice exposed to 4NQO alone (Group A) $(\mathrm{P}<0.05)$ (Figure 1D). Furthermore, significant differences in Ki-67, PCNA, and cyclin D1 expression levels were found at week 18 versus week 22 in the free-SAB and $4 \mathrm{NQO}$-treated groups but not the nanoSAB-treated mice (Figure 3D). These results indicate that nano-SAB may exert its chemopreventive effect of sustained drug release on oral carcinogenesis by maintaining low levels of Ki-67, PCNA, and cyclin D1 expression even after withdrawal of the drug for 4 weeks. Our previous in vitro study (8) demonstrated that the enhanced cellular uptake of nano-SAB could improve bioavailability by allowing sustained drug release in vivo. Accordingly, it can be inferred that nano-formulated $\mathrm{SAB}$ have superior efficacy for inhibiting proliferation and inducing cell cycle arrest, thereby improving the drug releasing profile of SAB. The efficacy of nano-SAB should be made with cautions because this was an in vivo experiment without human patients. Further studies are required to examine the mechanisms underlying the chemopreventive effects of nano-SAB on its anti-proliferative and sustained release responses in 4NQOinduced oral carcinogenesis.

In conclusion, the current in vivo study, together with our previous in vitro study, confirms that nano-SAB has superior efficacy of chemoprevention in promoting more potent anti-proliferation and cell cycle arrest responses via the blockade of Ki-67, PCNA, and cyclin D1 expression. Our study suggests the effect of nano-formulation on sustained drug release of SAB in 4NQO-induced oral carcinogenesis, further consolidating the potential of $\mathrm{SAB}$ nano-formulations as promising chemopreventive agents for treating oral carcinogenesis.

\section{Acknowledgments}

The authors thank International Science Editing for editing this manuscript.

Funding: This work was funded by National Natural Science Foundation of China (No. 82074502), the Science and Technology Commission of Shanghai Municipality (No. 21S21902000), the Cross-disciplinary Research Fund of Shanghai Ninth People's Hospital, Shanghai Jiao Tong University School of Medicine (No. JYJC202108), and the Fengxian District Clinical Diagnosis \& Treatment Center of Oral and Maxillofacial-Head and Neck Oncology (No. fxlczlzx-a-201705). 


\section{Footnote}

Reporting Checklist: The authors have completed the ARRIVE reporting checklist. Available at https://atm. amegroups.com/article/view/10.21037/atm-21-4457/rc

Data Sharing Statement: Available at https://atm.amegroups. com/article/view/10.21037/atm-21-4457/dss

Conflicts of Interest: All authors have completed the ICMJE uniform disclosure form (available at https://atm. amegroups.com/article/view/10.21037/atm-21-4457/coif). The authors have no conflicts of interest to declare.

Ethical Statement: The authors are accountable for all aspects of the work in ensuring that questions related to the accuracy or integrity of any part of the work are appropriately investigated and resolved. Experiments were performed under a project license (No. SH9H-2020-A85-1) granted by the Animal Ethical Committee of Shanghai Ninth People's Hospital, in compliance with internationally recognized and institutional guidelines for the care and use of animals.

Open Access Statement: This is an Open Access article distributed in accordance with the Creative Commons Attribution-NonCommercial-NoDerivs 4.0 International License (CC BY-NC-ND 4.0), which permits the noncommercial replication and distribution of the article with the strict proviso that no changes or edits are made and the original work is properly cited (including links to both the formal publication through the relevant DOI and the license). See: https://creativecommons.org/licenses/by-nc-nd/4.0/.

\section{References}

1. Miranda-Filho A, Bray F. Global patterns and trends in cancers of the lip, tongue and mouth. Oral Oncol 2020;102:104551.

2. Bosetti C, Carioli G, Santucci C, et al. Global trends in oral and pharyngeal cancer incidence and mortality. Int J Cancer 2020;147:1040-9.

3. Guimarães LM, Diniz MG, Rogatto SR, et al. The genetic basis of oral leukoplakia and its key role in understanding oral carcinogenesis. J Oral Pathol Med 2021;50:632-8.

4. Yap T, Celentano A, Seers C, et al. Molecular diagnostics in oral cancer and oral potentially malignant disorders-A clinician's guide. J Oral Pathol Med 2020;49:1-8.
5. Monteiro de Oliveira Novaes JA, William WN Jr. Prognostic factors, predictive markers and cancer biology: the triad for successful oral cancer chemoprevention. Future Oncol 2016;12:2379-86.

6. Crooker K, Aliani R, Ananth M, et al. A Review of Promising Natural Chemopreventive Agents for Head and Neck Cancer. Cancer Prev Res (Phila) 2018;11:441-50.

7. Lee TY, Tseng YH. The Potential of Phytochemicals in Oral Cancer Prevention and Therapy: A Review of the Evidence. Biomolecules 2020;10:1150.

8. Li H, Shi L, Wei J, et al. Cellular uptake and anticancer activity of salvianolic acid B phospholipid complex loaded nanoparticles in head and neck cancer and precancer cells. Colloids Surf B Biointerfaces 2016;147:65-72.

9. Chen YF, Chang KW, Yang IT, et al. Establishment of syngeneic murine model for oral cancer therapy. Oral Oncol 2019;95:194-201.

10. Ishida K, Tomita H, Nakashima T, et al. Current mouse models of oral squamous cell carcinoma: Genetic and chemically induced models. Oral Oncol 2017;73:16-20.

11. Luo JJ, Young CD, Zhou HM, et al. Mouse Models for Studying Oral Cancer: Impact in the Era of Cancer Immunotherapy. J Dent Res 2018;97:683-90.

12. Peng Q, Gong T, Zuo J, et al. Enhanced oral bioavailability of salvianolic acid B by phospholipid complex loaded nanoparticles. Pharmazie 2008;63:661-6.

13. Peng Q, Zhang ZR, Sun X, et al. Mechanisms of phospholipid complex loaded nanoparticles enhancing the oral bioavailability. Mol Pharm 2010;7:565-75.

14. Wei J, Wu J, Xu W, et al. Salvianolic acid B inhibits glycolysis in oral squamous cell carcinoma via targeting PI3K/AKT/HIF-1 $\alpha$ signaling pathway. Cell Death Dis 2018;9:599.

15. Wei J, Xie G, Ge S, et al. Metabolic transformation of DMBA-induced carcinogenesis and inhibitory effect of salvianolic acid b and breviscapine treatment. J Proteome Res 2012;11:1302-16.

16. Barnes L, Eveson JW, Reichart P, et al. World Health Organization classification of tumours. Pathology and genetics. Tumours of the oral cavity and oropharynx. Lyon: IARC Press, 2005:177-98.

17. Sequeira I, Rashid M, Tomás IM, et al. Genomic landscape and clonal architecture of mouse oral squamous cell carcinomas dictate tumour ecology. Nat Commun 2020;11:5671.

18. Sur S, Steele R, Aurora R, et al. Bitter Melon Prevents the Development of 4-NQO-Induced Oral Squamous Cell Carcinoma in an Immunocompetent Mouse Model by 
Modulating Immune Signaling. Cancer Prev Res (Phila) 2018;11:191-202.

19. Zargar-Shoshtari K, Sharma P, Spiess PE. Insight into novel biomarkers in penile cancer: Redefining the present and future treatment paradigm? Urol Oncol 2018;36:433-9.

20. Wang Y, Du G, Shi L, et al. Altered expression of CCN1 in oral lichen planus associated with keratinocyte activation and IL-1 $\beta$, ICAM1, and CCL5 up-regulation. J Oral Pathol Med 2020;49:920-5.

21. Hao Y, Xie T, Korotcov A, et al. Salvianolic acid B inhibits growth of head and neck squamous cell carcinoma in vitro and in vivo via cyclooxygenase- 2 and apoptotic pathways. Int J Cancer 2009; 124:2200-9.

22. Zhao Y, Hao Y, Ji H, et al. Combination effects of salvianolic acid B with low-dose celecoxib on inhibition of head and neck squamous cell carcinoma growth in vitro and in vivo. Cancer Prev Res (Phila) 2010;3:787-96.

23. Desai P, Thumma NJ, Wagh PR, et al. Cancer Chemoprevention Using Nanotechnology-Based Approaches. Front Pharmacol 2020;11:323.

24. Vijayalakshmi S, Mariadoss AVA, Ramachandran V, et al. Polydatin Encapsulated Poly [Lactic-co-glycolic acid]

Cite this article as: Liu W, Zhou Z, Zhu L, Li H, Wu L. Chemopreventive efficacy of salvianolic acid B phospholipid complex loaded nanoparticles against experimental oral carcinogenesis: implication of sustained drug release. Ann Transl Med 2022;10(5):244. doi: 10.21037/atm-21-4457
Nanoformulation Counteract the 7,12-Dimethylbenz[a] Anthracene Mediated Experimental Carcinogenesis through the Inhibition of Cell Proliferation. Antioxidants (Basel) 2019;8:375.

25. Mariadoss AVA, Vinayagam R, Xu B, et al. Phloretin loaded chitosan nanoparticles enhance the antioxidants and apoptotic mechanisms in DMBA induced experimental carcinogenesis. Chem Biol Interact 2019;308:11-9.

26. Dev A, Sardoiwala MN, Kushwaha AC, et al. Genistein nanoformulation promotes selective apoptosis in oral squamous cell carcinoma through repression of $3 \mathrm{PK}-$ EZH2 signalling pathway. Phytomedicine 2021;80:153386.

27. Li H, Qu X, Qian W, et al. Andrographolide-loaded solid lipid nanoparticles enhance anti-cancer activity against head and neck cancer and precancerous cells. Oral Dis 2022;28:142-9.

28. Mariadoss AVA, Vinayagam R, Senthilkumar V, et al. Phloretin loaded chitosan nanoparticles augments the $\mathrm{pH}$-dependent mitochondrial-mediated intrinsic apoptosis in human oral cancer cells. Int J Biol Macromol 2019;130:997-1008. 


\section{Supplementary}

Table S1 Bodyweight and histological charges of the experimental groups

\begin{tabular}{|c|c|c|c|}
\hline Group & Group A & Group B & Group C \\
\hline \multicolumn{4}{|l|}{ Bodyweight (mean, g) } \\
\hline Maximum (SD) & $27.41(1.73)$ & $27.09(1.61)$ & $25.76(1.19)$ \\
\hline Minimum (SD) & $18.36(0.99)$ & $18.91(0.63)$ & $19.05(1.55)$ \\
\hline Histological examination at $8 w(n)$ & 8 & 8 & 7 \\
\hline Normal & 4 & 6 & 7 \\
\hline Mild dysplasia & 2 & 2 & 0 \\
\hline Moderate/severe dysplasia & 2 & 0 & 0 \\
\hline Mild dysplasia & 2 & 3 & 4 \\
\hline Moderate/severe dysplasia & 5 & 4 & 3 \\
\hline Carcinoma & 1 & 0 & 0 \\
\hline Histological examination at $18 w(n)$ & 9 & 7 & 8 \\
\hline Mild dysplasia & 1 & 1 & 3 \\
\hline Moderate/severe dysplasia & 5 & 5 & 4 \\
\hline Carcinoma & 3 & 1 & 1 \\
\hline Cumulative incidence at $18 w(n)$ & 25 & 22 & 22 \\
\hline Moderate/severe dysplasia & 2 & 4 & 4 \\
\hline Carcinoma & 8 & 4 & 2 \\
\hline Cumulative incidence at $22 \mathrm{w}(\mathrm{n})$ & 35 & 30 & 30 \\
\hline No/mild dysplasia & 9 & 12 & 16 \\
\hline Moderate/severe dysplasia & 14 & 13 & 11 \\
\hline Carcinoma & 12 & 5 & 3 \\
\hline
\end{tabular}

SD, standard deviation; 4NQO, 4-nitroquinoline-N-oxide; SAB, salvianolic acid B; PLC-NP, phospholipid complex loaded nanoparticles. 